\title{
Overexpression of SREBP1 (sterol regulatory element binding protein 1) promotes de novo fatty acid synthesis and triacylglycerol accumulation in goat mammary epithelial cells
}

\author{
H. F. Xu, ${ }^{*}$ J. Luo, ${ }^{* 1}$ W. S. Zhao, ${ }^{*}$ Y. C. Yang, ${ }^{*}$ H. B. Tian, ${ }^{*}$ H. B. Shi, ${ }^{*}$ and M. Bionaz ${ }^{1}$ \\ *Shaanxi Key Laboratory of Molecular Biology for Agriculture, College of Animal Science and Technology, Northwest A\&F University, \\ Yangling, 712100, China \\ †Department of Animal and Rangeland Sciences, Oregon State University, Corvallis 97331
}

\begin{abstract}
Sterol regulatory element binding protein 1 (SREBP1; gene name $S R E B F 1$ ) is known to be the master regulator of lipid homeostasis in mammals, including milk fat synthesis. The major role of SREBP1 in controlling milk fat synthesis has been demonstrated in bovine mammary epithelial cells. Except for a demonstrated role in controlling the expression of FASN, a regulatory role of SREBP1 on milk fat synthesis is very likely, but has not yet been demonstrated in goat mammary epithelial cells (GMEC). To explore the regulatory function of SREBP1 on de novo fatty acids and triacylglycerol synthesis in GMEC, we overexpressed the mature form of SREBP1 (active $\mathrm{NH}_{2}$-terminal fragment) in GMEC using a recombinant adenovirus vector (AdnSREBP1), with Ad-GFP (recombinant adenovirus of green fluorescent protein) as control, and infected the GMEC for $48 \mathrm{~h}$. In infected cells, we assessed the expression of 20 genes related to milk fat synthesis using real time-quantitative PCR, the protein abundance of SREBP1 and FASN by Western blot, the production of triacylglycerol, and the fatty acid profile. Expression of $S R E B F 1$ was modest in mammary compared with the other tissues in dairy goats but its expression increased approximately 30-fold from pregnancy to lactation. The overexpression of the mature form of SREBP1 was confirmed by $>200$-fold higher expression of SREBF1 in Ad-nSREBP1 compared with Ad-GFP. We observed no changes in amount of the precursor form of SREBP1 protein but a $>10$-fold increase of the mature form of SREBP1 protein with Ad-nSREBP1. Compared with Ad-GFP cells (control), Ad-nSREBP1 cells had a significant increase in expression of genes related to long-chain fatty acid activation (ACSL1), transport
\end{abstract}

Received April 22, 2015

Accepted September 22, 2015.

${ }^{1}$ Corresponding authors: luojun@nwsuaf.edu.cn and massimo. bionaz@oregonstate.edu
(FABP3), desaturation (SCD1), de novo synthesis of fatty acids (ACSS2, ACLY, IDH1, ACACA, FASN, and ELOVL6), and transcriptional factors (NR1H3 and $P P A R G$ ). We observed a $>10$-fold increase in expression of INSIG1 but SCAP was downregulated by AdnSREBP1. Among genes related to milk fat synthesis and lipid droplet formation, only LPIN1 and DGAT1 were upregulated by Ad-nSREBP1. Compared with the Ad-GFP, the cellular triacylglycerol content was higher and the percentage of $\mathrm{C} 16: 0$ and $\mathrm{C} 18: 1$ increased, whereas that of $\mathrm{C} 16: 1, \mathrm{C} 18: 0$, and $\mathrm{C} 18: 2$ decreased in Ad-nSREBP1 cells. Overall, the data provide strong support for a central role of SREBP1 in the regulation of milk fat synthesis in goat mammary cells.

Key words: sterol regulatory element binding protein 1 (SREBP1), milk fat synthesis, adenovirus, goat

\section{INTRODUCTION}

The sterol regulatory element binding protein 1 (SREBP1) belongs to the basic helix-loop-helix leucine zipper transcription factor superfamily (Yokoyama et al., 1993). Two isoforms of SREBP1 (gene name $S R E B F 1$ ) have been identified in mammals, SREBP1a and SREBP-1c, which differ by use of alternative promoters (Horton, 2002). The SREBP isoforms are synthesized as inactive endoplasmic reticulum (ER) membrane-bound protein precursors (Tontonoz et al., 1993; Brown and Goldstein, 1997). Under low sterol concentrations, SREBP cleavage activating protein (SCAP) escorts the SREBP precursors from ER to Golgi apparatus, where SREBP precursors are cleaved to release the N-terminal active fragment (Brown and Goldstein, 1997; Espenshade, 2006; Sato, 2010). The active fragment enters into the nucleus, where it binds the SREBP response element (SRE) in the promoter region of many lipid metabolism-related genes to increase their transcription (Horton, 2002; Eberlé et al., 2004). Under high intracellular sterol concentrations, insulin-induced gene (INSIG) is activated and binds 
SCAP. The SREBP-SCAP-INSIG complex is retained in the ER membrane, inhibiting the activity of SREBP1 (Nohturfft et al., 1998).

The synthesis of milk fat involves many proteins including transporters and enzymes (Moore and Christie, 1979; Clegg et al., 2001). A model summarizing all main proteins involved in milk fat synthesis was previously proposed (Bionaz and Loor, 2008b). Central to the transcriptional regulation of milk fat synthesis is SREBP1, as suggested by studies performed in bovine (Harvatine and Bauman, 2006; Ma and Corl, 2012; Oppi-Williams et al., 2013) and mouse (Rudolph et al., 2010). Recently, it was demonstrated in bovine mammary epithelial cells by gene overexpression and knockdown that SREBP1 is central in transcription regulation of many genes related to milk fat synthesis and secretion ( $\mathrm{Li}$ et al., 2014a).

Compared with rodents, humans, and bovines, much less is known about the role of SREBP1 in the regulation of milk fat synthesis in mammary epithelial cells of dairy goats. An initial suggestion of a role of SREBP1 in milk fat synthesis in goat was provided by a very recent study, where overexpression of $S R E B F 1$ increased the expression of FASN and $S R E B F 1$ in primary goat mammary epithelial cells (Li et al., 2015); however, no expression of additional genes or synthesis of triacylglycerol (TAG) was assessed in that study.

The objective of the present study was to examine the role of SREBP1 in the regulation of milk fat synthesis in goat. To accomplish our objective, we overexpressed the mature form of SREBP1 (i.e., active $\mathrm{NH}_{2}$-terminal fragment) in primary goat mammary epithelial cells (GMEC) and assessed the effect on expression of a large number of milk fat-related genes and the effect on cellular fatty acid profile and TAG content.

\section{MATERIALS AND METHODS}

All experimental procedures involving dairy goats were conducted under the approval of the Animal Care and Use Committee of Northwest A\&F University of Agriculture and Technology (Yangling, Shaanxi, China).

\section{Tissue Sample Collection}

Three-year-old Xinong Saanen dairy goats of Northwest $A \& F$ University experimental farm were selected and killed for mammary gland tissue collection during lactation (120 d after parturition, $\mathrm{n}=3$ ) and the dry period (10 d after dry-off and $50 \mathrm{~d}$ before expected parturition, $\mathrm{n}=3$ ). Samples were washed with diethylpyrocarbonate (DEPC)-treated water and then immediately snap-frozen in liquid nitrogen.

\section{Generation of Recombinant Adenoviruses}

The transcriptionally active $\mathrm{NH}_{2}$-terminal fragment (AA 1-403) of goat SREBP1 was amplified and used to generate recombinant adenovirus according to previously published reports (He et al., 1998; Shi et al., 2014). Briefly, the cDNA length of 1-1,209 nucleotides of SREBP1 (nSREBP1) was amplified and subcloned into the shuttle vector pAdTrack-CMV. The recombinant plasmid pAdTrack-CMV-nSREBP1 linearized by restriction endonuclease PmeI was transformed into Escherichia coli BJ5183 competent cells containing the backbone vector pAdEasy-1. Homologous recombination occurred between shuttle vector and backbone vector. Positive recombinants of plasmid (pAd-nSREBP1) were identified by kanamycin resistance and digestion of restriction endonuclease PacI. Then, the linearized product of pAd-nSREBP1 digested by PacI was transfected into HEK293 cells to amplify high-titer recombinant adenovirus (Ad-nSREBP1). The recombinant adenovirus of green fluorescent protein (GFP) was used as control (Ad-GFP). Detailed procedures on purification, titer determination, and storing conditions of adenovirus were previously provided (Shi et al., 2013a).

\section{Cell Culture and Treatments}

The HEK293 cells were cultured in basal RPMI1640 medium (Hyclone Laboratories, Beijing, China) supplemented with $10 \%$ fetal bovine serum (Hyclone) and penicillin/streptomycin $(10 \mathrm{kU} / \mathrm{L}$, Harbin Pharmaceutical Group, Harbin, China). Cells were cultured at $37^{\circ} \mathrm{C}$ in $5 \% \mathrm{CO}_{2}$ and medium was changed every $24 \mathrm{~h}$.

The GMEC were isolated, purified, and cultured from 3 lactating goats according to our previously described protocol (Shi et al., 2013a). Isolated cells were maintained in a basal Dulbecco's modified Eagle medium/F12 medium (Hyclone) supplemented with 10\% fetal bovine serum (Hyclone), bovine insulin $(5 \mu \mathrm{g} / \mathrm{mL}$; Sigma, St. Louis, MO), hydrocortisone ( $5 \mathrm{mg} / \mathrm{L}$; Sigma), penicillin/streptomycin (10 kU/L; Harbin Pharmaceutical Group), and epidermal growth factor (10 $\mathrm{ng} / \mathrm{mL}$; Sigma). The medium contained an estimated $315 \mathrm{mg} / \mathrm{dL}$ of glucose (i.e., a supraphysiological level in goat, where the norm is between 40 and $75 \mathrm{mg}$ of glucose/dL in blood) and did not contain acetate, with the exception of a negligible dose provided through the fetal bovine serum. Cells were incubated at $37^{\circ} \mathrm{C}$ in $5 \% \mathrm{CO}_{2}$ and culture medium was changed every $24 \mathrm{~h}$. The GMEC isolated from the 3 goats were cultured individually to passage 5 and pooled before starting the experiment. For RNA extraction, cells were seeded in 6-well cell culture plate (Corning Inc., Corning, NY). For protein extraction and quantification of total 
cellular TAG, cells were seeded in $60-\mathrm{mm}$ cell culture dishes (Corning Inc.). Once the culture reached 80 to 90\% confluence, cells were washed twice with PBS, and Ad-nSREBP1 or Ad-GFP recombinant adenovirus was added to the cells with the basal culture medium supplemented with prolactin $(1.5 \mu \mathrm{g} / \mathrm{mL}$; Sigma $)$. Cells were harvested after $48 \mathrm{~h}$ of incubation. Each treatment was performed in 3 replicates.

\section{RNA Extraction and Real-Time Quantitative PCR}

Total RNA was extracted from tissue and cells using RNAprep pure cell/bacteria kit (Tiangen Biotech Co. Ltd., Beijing, China) according to the manufacturer's instructions. The purity and concentration of RNA were determined by using a NanoDrop 2000 spectrophotometer (Thermo Fisher Scientific, Waltham, MA), the ratio of optical density at $260 \mathrm{~nm}: 280 \mathrm{~nm}$ was $\geq 2$ for all samples. Integrity of RNA was assessed by electrophoretic analysis of $28 \mathrm{~S}$ and $18 \mathrm{~S}$ rRNA subunits stained with ethidium bromide. Results were analyzed by Gel-Pro Analyzer software (Beijing JunyiDongfang Electrophoresis Equipment Co. Ltd., Beijing, China) and the ratio of $28 \mathrm{~S}: 18 \mathrm{~S}$ was approximately $2: 1$ (Supplemental Figure S1; http://dx.doi.org/10.3168/ jds.2015-9736). For the analysis of mRNA abundance of SREBF1 between tissues, the same amount of RNA extracted from each type of tissue was pooled from the 3 goats before cDNA synthesis. For mammary tissue during the 2 stages of lactation and for GMEC, cDNA was synthesized from each individual extracted sample. First-strand cDNA was synthesized $(0.5 \mu \mathrm{g}$ of RNA per $10-\mu \mathrm{L}$ reaction) using PrimeScript RT reagent Kit (Takara Bio Inc., Otsu, Japan) according to the manufacturer's instructions. Real-time quantitative PCR (RT-qPCR) was performed using SYBR Premix Ex Taq II Kit (Takara Bio Inc.) on a CFX96 Real-Time PCR Detection system (Bio-Rad Laboratories Inc., Hercules, CA). The amplification conditions were as follows: 1 cycle at $95^{\circ} \mathrm{C}$ for $30 \mathrm{~s}$, followed by 39 cycles at $95^{\circ} \mathrm{C}$ for $5 \mathrm{~s}$, and $60^{\circ} \mathrm{C}$ for $30 \mathrm{~s}$; a dissociation curve of each sample was generated at $95^{\circ} \mathrm{C}$ for $10 \mathrm{~s}$ and then from $65^{\circ} \mathrm{C}$ to $95^{\circ} \mathrm{C}$ with an increment of $0.5^{\circ} \mathrm{C}$. Gene-specific primers for $S R E B F 1$ between tissues and different lactating stages were as follows: 5'-GCTGAGTGCCCTGAACC-3' and 5'-CCATCCACGAAGAAACG-3' (i.e., spanning exon 11 to 12, common to all $S R E B F 1$ isoforms). The primer pair for the internal control, GAPDH, was 5'-GCAAGTTCCACGGCACAG-3' and 5'-GGTTCACGCCCATCACAA-3'. Gene-specific primers to assess expression of genes in GMEC were designed using Primer Premier 5 (Premier Biosoft, Palo Alto, CA; Table 1). Two primer pairs were designed for $S R E B F 1$, one to specifically amplify SREBF1a transcript (spanning the exon 1, present only in isoform SREBP-1a) and one to measure simultaneously all $S R E B F 1$ isoforms (spanning exon 3, common to all SREBF1 isoforms). All primers were tested for presence of a single band at the expected size using a $2 \%$ agarose gel and amplicons were sequenced according to methods described previously (Kadegowda et al., 2009; Shi et al., 2013a).

The mRNA abundance of SREBF1 between tissues and in mammary tissue in the 2 different lactation stages was determined using the $2^{\Delta \Delta \mathrm{Cq}}$ method, where $\mathrm{Cq}$ is the quantitation cycle, and GAPDH was used as internal control. For the measurement of gene expression in GMEC, the RDML (real-time PCR data markup language; http://www.rdml.org/) files were exported and uploaded into the LinRegPCR software (www.linregpcr.nl) for quantification analysis (Ramakers et al., 2003). The normalization factor was calculated as the geometrical mean of the RT-qPCR data obtained from LinRegPCR analysis of mitochondrial ribosomal protein L39 (MRPL39), ribosomal protein S9 (RSP9), and ubiquitously expressed transcript $(U X T)$. The stability of the internal control genes and the reliability of the calculated normalization factor were evaluated using geNorm (Vandesompele et al., 2002). The M-value (expression stability) was $<0.28$ for all 3 internal control genes and the $\mathrm{V}$-value (pairwise variation) was 0.11 ; thus, all 3 internal control were very stable and the normalization factor reliable according to the threshold suggested by Vandesompele and collaborators (2002; i.e., $\leq 1.5$ for $M$-value and $\leq 0.15$ for $\mathrm{V}$-value). The final value of each target gene was obtained by dividing the RT-qPCR data obtained from LinRegPCR analysis by the normalization factor. The percentage RNA abundance of each genes relative to SREBF1a was calculated as $\left[\mathrm{E}^{-\mathrm{Cq} \text { gene }} / \mathrm{E}^{-\mathrm{Cq} S R E B F 1 a}\right]$ $\times$ 100. The efficiency $(\mathrm{E})$ of PCR amplification was obtained using LinRegPCR (Table 1).

\section{Protein Extraction and Western Blot}

Cells were washed twice with cold PBS before harvest. Total protein was extracted with radioimmunoprecipitation assay (RIPA) buffer (Solarbio Tech Co. Ltd., Beijing, China) supplemented with phenylmethanesulfonyl fluoride (PMSF; Pierce, Rockford, IL) at a final concentration of $1 \mathrm{mM}$. The protein concentration was measured using a bicinchoninic acid (BCA) kit (Thermo Fisher Scientific) according to the manufacturer's instruction, and $25 \mu \mathrm{g}$ of total protein per lane was separated by SDS-PAGE. The blot was transferred to nitrocellulose membrane (Pall Corp., Port Washing- 


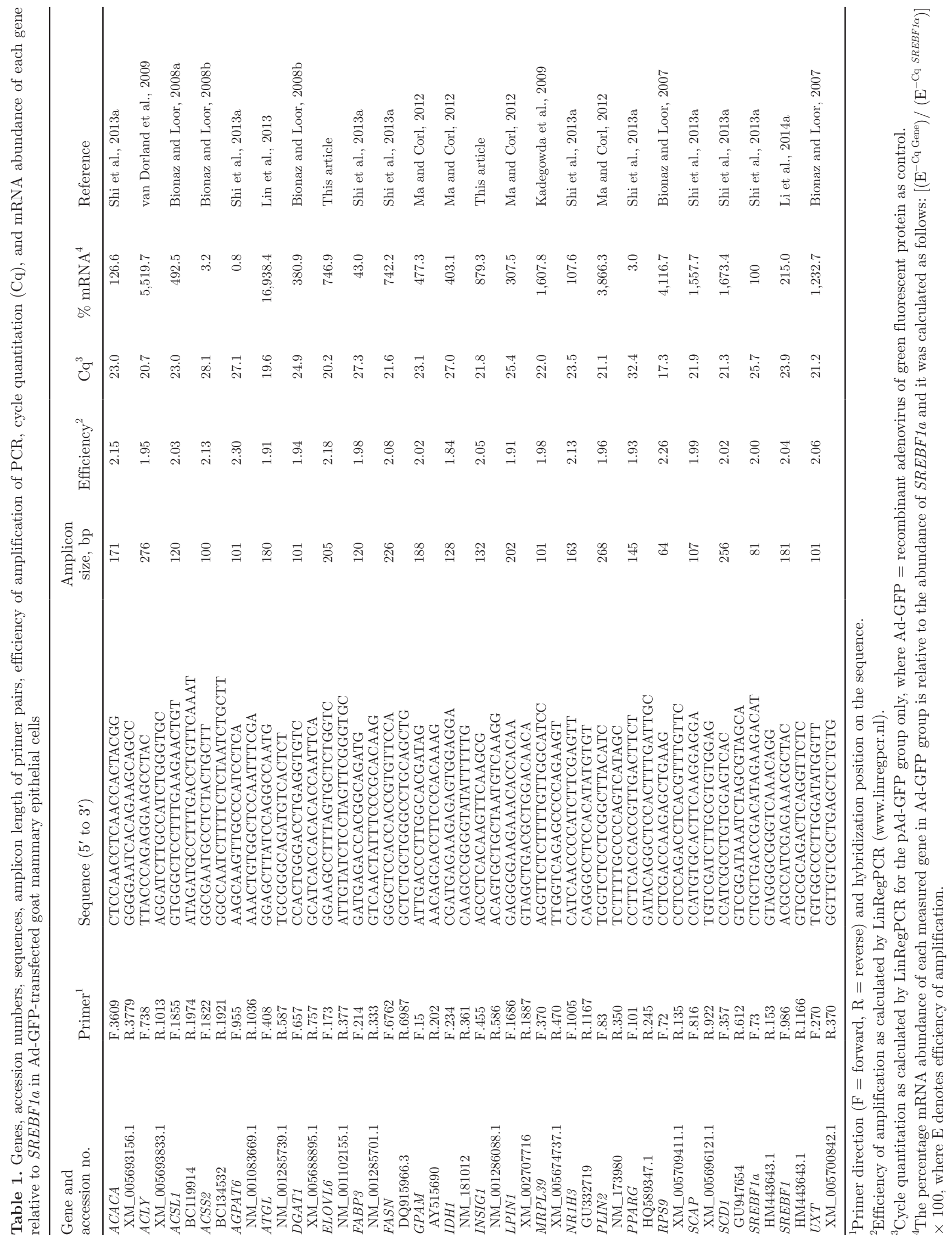


ton, NY) by a Bio-Rad Trans-Blot SD semi-dry transfer cell (Bio-Rad Laboratories Inc.). Membranes were blocked for $1 \mathrm{~h}$ using $5 \%$ skim milk in Tris-buffered saline/Tween-20 (150 mM NaCl, $10 \mathrm{~m} M$ Tris-HCl, pH $7.5,0.1 \%$ Tween-20) before incubation overnight at $4^{\circ} \mathrm{C}$ with primary antibodies for $\beta$-actin (CW0096, 1:1,000; CW Biotech, Beijing, China), SREBP1 (ab3259, 1:500; Abcam, Cambridge, UK), and FASN (1:300; Li et al., 2014a). Horseradish peroxidase (HRP)-conjugated goat anti-mouse (CW0102, 1:1,000; CW Biotech) and goat anti-rabbit IgG (CW0103, 1:1,000; CW Biotech) were used as secondary antibodies. Signal was measured using the chemiluminescent ECL Western blot detection system (Thermo). The grayscale data of each band were analyzed using ImageJ software (http://imagej. nih.gov/ij/) and relative protein abundance was normalized to $\beta$-actin.

\section{Measurement of Total Cellular TAG}

After $48 \mathrm{~h}$ of incubation with adenovirus, cells were rinsed twice with cold PBS and the total content of cellular TAG was extracted by using a tissue/cell triacylglycerol assay kit, according to the manufacturer's instruction (Applygen Technologies Inc., Beijing, China), and the data were read on a microtiter plate reader (Bio-Rad Laboratories Inc.). Total TAG content was normalized to total cellular protein quantified by using a bicinchoninic acid protein assay kit (Thermo Fisher Scientific).

\section{Measurement of Fatty Acid in GMEC}

Total lipid was extracted from $60-\mathrm{mm}$ culture dish of GMEC using $2 \mathrm{~mL}$ of $2.5 \%$ ( $\mathrm{vol} / \mathrm{vol}$ ) sulfuric acid:methanol (Sigma) in an 8-mL glass tube. After ultrasonication for $10 \mathrm{~min}$, the glass tubes were incubated at $80^{\circ} \mathrm{C}$ for $1 \mathrm{~h}$. After cooling the tube to room temperature, $2 \mathrm{~mL}$ of $0.1 \mathrm{M} \mathrm{HCl}$ and $800 \mu \mathrm{L}$ of hexyl hydride (Sigma) were added and the tube was vortexed for $30 \mathrm{~s}$ followed by centrifugation for $5 \mathrm{~min}$ at $900 \times$ g. Supernatant was collected in a $2-\mathrm{mL}$ silicified tube with $0.5 \mathrm{~g}$ of water-free sodium sulfate. After acute shock, the tube was centrifuged for $3 \mathrm{~min}$ at $13,800 \times g$, and the liquid supernatant was collected for fatty acid analysis by GC-MS (Thermo Fisher Trace GC ultraDSQII-AI3000) using an BP-5MS $(30 \mathrm{~m} \times 0.25 \mathrm{~mm} \times$ $0.25 \mu \mathrm{m}$ ) column (Agilent Technologies, Santa Clara, CA). A commercial C19:0 fatty acid (Sigma) was used as an internal control. The relative proportion of each fatty acid was determined as percentage of the total peak area that could be identified (Wang et al., 2012; Shi et al., 2013b).

\section{Statistical Analysis}

For GMEC, normalized RT-qPCR data were divided by the average of the control group, and transformed data were checked for normal distribution using Univariate Proc of SAS (version 9.4, SAS Institute Inc., Cary, NC) before statistical analysis. This was performed using GLM of SAS with treatment (Ad-nSREBP1 and Ad-GFP) or tissue as main effect and replicates as random. Least squares means (LSM) were separated using Tukey's test. Differences were considered statistically significant when $P<0.05$, and data are reported as $\mathrm{LSM} \pm \mathrm{SE}$.

\section{RESULTS}

\section{Transcript Abundance of SREBF1 in Mammary of Dairy Goats in Pregnancy and Lactation}

The raw RT-qPCR data of GAPDH were not different between nonlactating (i.e., pregnant) and lactating mammary tissue in goat (data not shown), and the RT-qPCR data of SREBF1 normalized with GAPDH revealed a $\sim 30$-fold increase in expression from pregnancy to lactation (see insert in Supplemental Figure S2A; http://dx.doi.org/10.3168/jds.2015-9736). Overall, mammary tissue had an intermediate mRNA abundance of SREBF1 (Supplemental Figure S2; http:// dx.doi.org/10.3168/jds.2015-9736). Abundance of SREBF1 between tissues is reported in Supplemental Figure S2 (http://dx.doi.org/10.3168/jds.2015-9736).

\section{Ad-SREBP1 Overexpresses Mature SREBP1 in GMEC}

The mRNA abundance of SREBF1a increased $>300$ fold and SREBF1 increased $>250$-fold in Ad-nSREBP1infected cells compared with Ad-GFP-infected cells (Figure 1A). Overexpression of SREBF1 transcriptionally active $\mathrm{NH}_{2}$-terminal fragment (nSREBP1) led to a $>10$-fold increase of SREBP1 mature protein, whereas the precursor form of SREBP1 protein was not affected (Figure 1B).

\section{Overexpression of SREBF1 Promotes Expression of Lipogenic Genes and Increases Cellular TAG}

Compared with Ad-GFP infected cells, overexpression n-SREBP1 dramatically upregulated the mRNA levels of all measured genes coding for proteins involved in fatty acid activation, transport, and desaturation, encompassing fatty acid binding protein 3 (FABP3; $>100$-fold), acyl-CoA synthase long-chain family member 1 (ACSL1; $\sim 4$-fold), ELOVL fatty acid elongase 6 (ELOVL6; >2-fold), and stearoyl-CoA desaturase 
(SCD1; >6-fold; Figure 2). Overexpression of nSREBP1 increased the transcription of all genes measured involved in de novo fatty acid synthesis, with $>50$-fold increase for acyl-CoA synthetase short-chain family member 2 (ACSS2), >9-fold increase for fatty acid synthase $(F A S N),>2$-fold increase for acetyl-CoA carboxylase $\alpha(A C A C A),>1.5$-fold increase for isocitrate dehydrogenase $1(\mathrm{NADP}+)$ soluble $(I D H 1)$, and $\sim 6$-fold increase for ATP citrate lyase $(A C L Y)$. The increase in expression of $F A S N$ was also confirmed by the increase in its protein abundance (Figure 2; Supplemental Table S1; http://dx.doi.org/10.3168/jds.2015-9736). The expression of most of the transcription regulation-related genes, including INSIG1, NR1H3, and PPARG, was upregulated by overexpression of nSREBP1 but the expression of $S C A P$ was downregulated (Figure 2).

Few genes coding for proteins related to TAG synthesis and lipid droplet formation were affected by over-
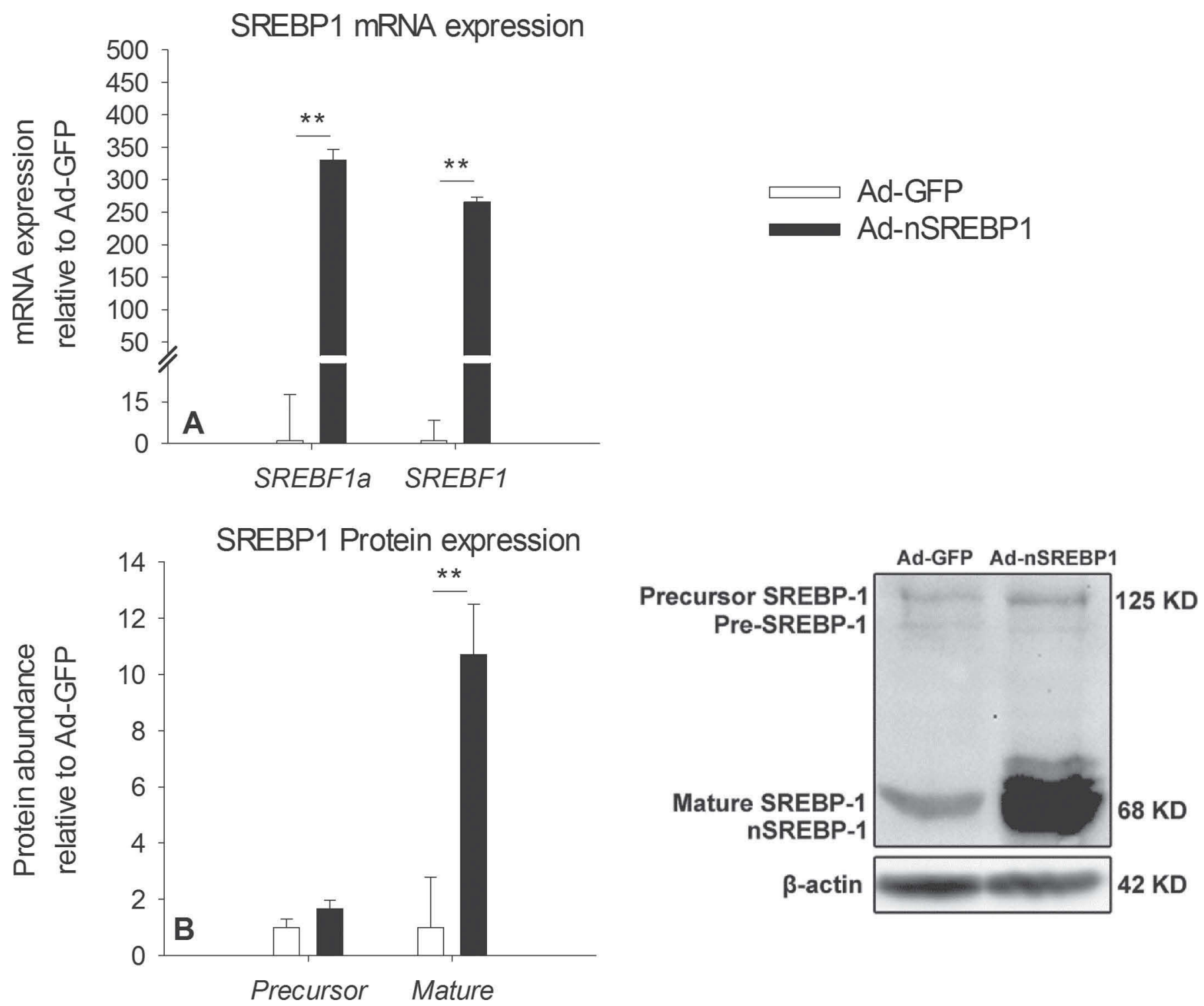

Figure 1. Adenovirus-mediated overexpression of nSREBP1 (the transcriptionally active $\mathrm{NH}_{2}$-terminal fragment of the sterol regulatory element binding protein 1 gene, SREBF1) in primary goat mammary epithelial cells. (A) Real-time quantitative PCR analysis of SREBF1 expression after $48 \mathrm{~h}$ of infection with Ad-nSREBP1 adenovirus. Values are presented as LSM \pm SE for 3 replicates; $* * P<0.01$. (B) Western blot analysis of precursor $(125 \mathrm{kDa})$ and mature $(68 \mathrm{kDa})$ SREBP1 protein levels after $48 \mathrm{~h}$ infection with Ad-nSREBP1 adenovirus. Protein abundance was normalized to $\beta$-actin. The blots are representative of 3 independent experiments. 
expression of nSREBP1 (Figure 3). Among these were lipin 1 (LPIN1), which increased $>15$-fold, and diacylglycerol O-acyltransferase 1 (DGAT1), which increased $>1.5$-fold. No effects were detected for 2 of the enzymes involved in TAG synthesis: glycerol-3-phosphate acyltransferase 1 (GPAM) and 1-acylglycerol-3-phosphate O-acyltransferase 6 (AGPAT6), or for genes coding for perilipin 2 (PLIN2), involved in lipid droplet formation, and adipose triacylglycerol lipase $(A T G L)$, responsible for the lipid droplet TAG depletion (Figure 3).

\section{Overexpression of SREBF1 Affects Fatty Acid Composition in GMEC}

Because of increased expression of TAG synthesisrelated genes, total cellular content of TAG also increased in cells transfected with Ad-nSREBP1 (Figure 3). The fatty acid profile in GMEC was affected with an increase in the proportion of $\mathrm{C} 16: 0$, total C18:1, and cis-9 $\mathrm{C} 18: 1$ and a decrease in proportion of $\mathrm{C} 16: 1$, C18:0, and C18:2, whereas relative abundance of 2

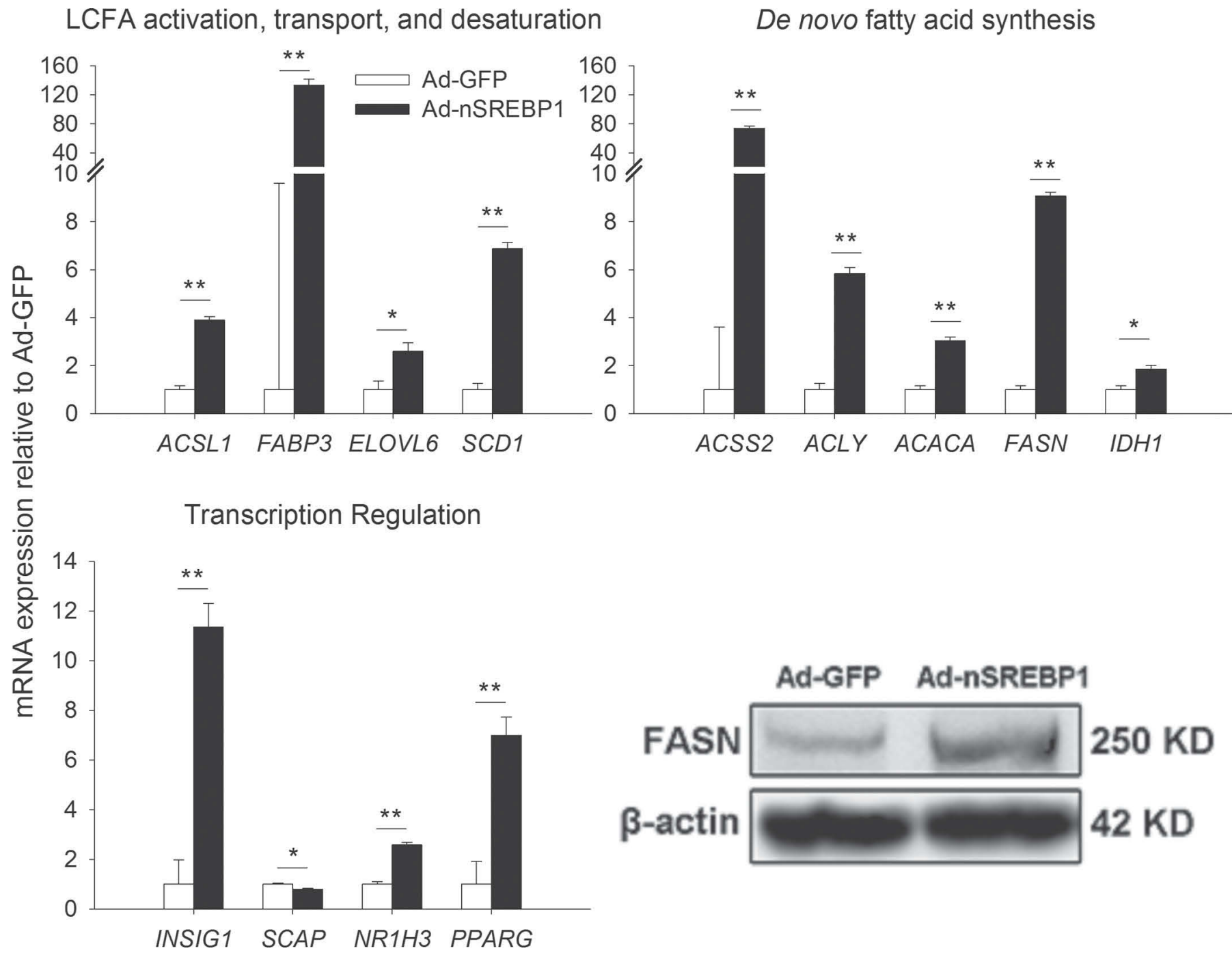

Figure 2. Adenovirus-mediated overexpression of nSREBP1 (the transcriptionally active $\mathrm{NH}_{2}$-terminal fragment of the sterol regulatory element binding protein 1 gene, $S R E B F 1)$ upregulates the expression of genes related to fatty acid synthesis in goat mammary epithelial cells Reported is the effect of nSREBP1 overexpression on main genes coding for proteins with key roles in long-chain fatty acid activation (ACSL1), transport (FABP3), desaturation (SCD1), de novo fatty acid synthesis (FASN, ACACA, ACSS2, and IDH1), regulation of SREBP1 activity (INSIG1 and SCAP), and transcriptional factors important for regulation of milk fat synthesis (i.e., PPARG and NR1H3). Expression of genes with unclear or not yet demonstrated role in mammary fatty acid synthesis (ACLY and ELOVL6) are also reported. The effect of nSREBP1 overexpression on the protein level of fatty acid synthase $(F A S N)$ is shown. Real-time quantitative PCR measurements of gene expression are expressed as fold change compared with Ad-GFP-transfected (recombinant adenovirus of green fluorescent protein as control) cells. Values are presented as $\mathrm{LSM} \pm \mathrm{SE}$ for 3 replicates; ${ }^{* *} P<0.01$. 
Table 2. Effects of overexpression of nSREBP1 (transcriptionally active $\mathrm{NH}_{2}$-terminal fragment of the sterol regulatory element binding protein 1 gene, SREBF1) on fatty acid composition (\%; means \pm SD) in goat mammary epithelial cells ${ }^{1}$

\begin{tabular}{lrrr}
\hline Fatty acid & \multicolumn{1}{c}{ Ad-GFP } & Ad-nSREBP1 & P-value \\
\hline C16:0 & $22.40 \pm 0.70$ & $25.29 \pm 0.53$ & 0.042 \\
cis-11 C16:1 + trans-9 C16:1 & $1.69 \pm 0.38$ & $0.79 \pm 0.04$ & 0.047 \\
C18:0 & $19.04 \pm 0.69$ & $12.5 \pm 0.60$ & 0.004 \\
C18:1 (total) & $37.85 \pm 0.72$ & $43.79 \pm 0.57$ & 0.006 \\
cis-9 C18:1 & $32.03 \pm 0.88$ & $37.50 \pm 1.26$ & 0.043 \\
C18:2 & $2.78 \pm 0.25$ & $1.49 \pm 0.10$ & 0.006 \\
C20:4 & $8.90 \pm 0.89$ & $8.82 \pm 0.31$ & 0.923 \\
C22:6 & $7.34 \pm 0.06$ & $7.29 \pm 0.03$ & 0.481 \\
\hline
\end{tabular}

${ }^{1}$ Ad-GFP $=$ recombinant adenovirus of green fluorescent protein as control; Ad-nSREBP1 = adenovirusmediated overexpression of the transcriptionally active $\mathrm{NH}_{2}$-terminal fragment of the sterol regulatory element binding protein 1 gene.

PUFA, C20:4 and C22:6, was not affected (Table 2; see Supplemental Figures S3 and S4 for examples of chromatographs; http://dx.doi.org/10.3168/jds.2015-9736).

\section{DISCUSSION}

\section{Expression of SREBF1 Between Physiological States}

The SREBP proteins were first identified and isolated from nuclear extracts of cultured human HeLa cells in 1993 (Briggs et al., 1993; Wang et al., 1993; Brown and Goldstein, 1997). The gene expression profile of SREBP-1a and SREBP-1c is different, as observed in mice and humans, where the $S R E B P-1 c$ gene is highly expressed in the liver, white adipose tissue, skeletal muscle, adrenal gland, and brain, whereas SREBP$1 a$ is highly expressed in cell lines and tissues with high capacity for cell proliferation, such as spleen and intestine (Desvergne et al., 2006; Chen et al., 2013). Relative mRNA abundance of SREBF1 between tissues is reported in the Supplemental File (http://dx.doi. org/10.3168/jds.2015-9736).

The large increase in expression of SREBF1 from pregnancy to lactation in mammary tissue of goats confirmed the previously observed increase in expression of this gene in mammary tissue of other species in the same physiological changes, such as in murine (Rudolph et al., 2010) and bovine (Bionaz and Loor, 2008b), but

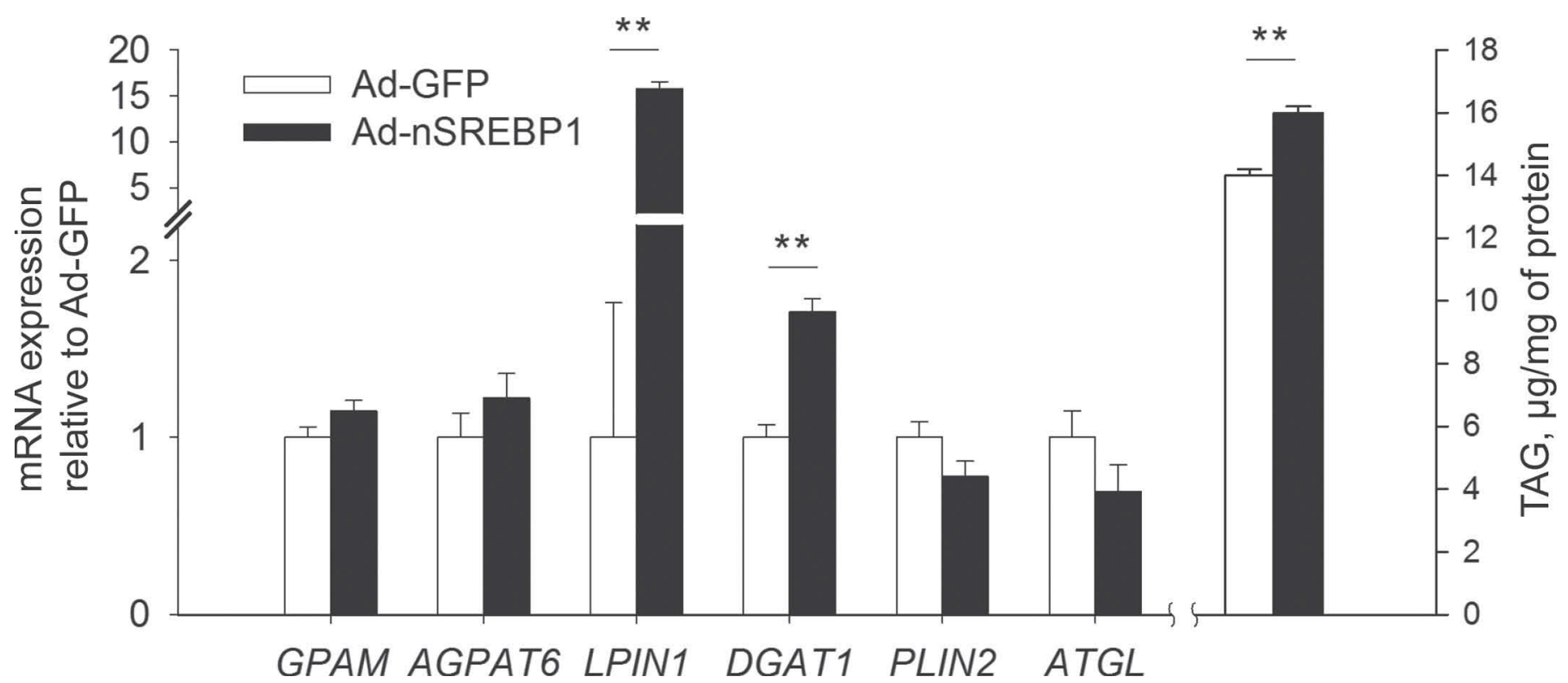

Figure 3. Adenovirus-mediated overexpression of nSREBP1 (the transcriptionally active $\mathrm{NH}_{2}$-terminal fragment of the sterol regulatory element binding protein 1 gene, SREBF1) increases cellular triacylglycerol content and expression of related genes in primary goat mammary epithelial cells (GMEC). Reported is the effect of nSREBP1 overexpression on main genes coding for proteins with key roles in triacylglycerol synthesis (LPIN1, GPAM, AGPAT6, and DGAT1) and lipid droplet formation (PLIN2 and ATGL). The amount of triacylglycerol (TAG) in GMEC is also shown. Values are presented as LSM \pm SE for 3 replicates; ${ }^{* *} P<0.01$. 
the magnitude of increase was much larger than that in other species $(\sim 2$-fold for mouse and bovine vs. $\sim 30$ fold for goat). The high expression of SREBF1 during lactation is thought to be related to the increased milk fat synthesis in sheep, bovine, and mouse (Bauman et al., 2006; Rudolph et al., 2010; Carcangiu et al., 2013), and SREBP1 is considered a central hub in the transcriptional network controlling milk fat synthesis in bovine (Bionaz and Loor, 2008b). This role has been previously suggested by investigation of the mechanism behind milk fat depression, which is partly caused by trans-10,cis-12 CLA. Conjugated linoleic acid depresses milk fat synthesis by decreasing the expression of milk fat synthesis-related genes via inhibition of SREBP1 activity and expression (Peterson et al., 2004; Bauman et al., 2008; Harvatine et al., 2009; Ma and Corl, 2012). Recent studies have established that SREBP1 is a central player in the regulation of milk fat synthesis in bovine (Lengi and Corl, 2010; Ma and Corl, 2012; Li et al., 2014a). Taken together, these results strongly support a key role of SREBP1 in milk fat synthesis in bovine. Initial evidence for a similar role in goats was provided by a recent study in which overexpression of SREBP1 increased the expression of FASN in GMEC (Li et al., 2015). The very large increase in expression from pregnancy to lactation in mammary tissue in our experiment provides additional support for a key role for SREBP1 in goats.

\section{Overexpression of nSREBP1 Increases Expression of SREBF1}

In this study, adenovirus-mediated overexpression technology was used to successfully overexpress the mature form of SREBP1, which is the functional form entering the cell nucleus and increasing the expression of its target genes. The successful overexpression was demonstrated by $>10$-fold increase in protein abundance of the mature form of SREBP1 and $>250$-fold increase in the expression of $S R E B F 1$. The primer pair used in the present experiment to amplify $S R E B F 1 a$ by RT-qPCR spans nucleotides 73 to 153 of the SREBP1 mRNA. This segment codes for positions 24 to 50 of the AA sequence of the SREBP1a protein, as estimated using data from a previous study in bovine (Lengi and Corl, 2010). Thus, we designed a primer pair that amplifies only the gene for $S R E B F 1 a$ and one primer pair that amplifies all $S R E B F 1$ isoforms. We did not design primer pairs to specifically measure SREBP-1c transcript. The overexpression of nSREBP1 increased the expression of $S R E B F 1 a>300$-fold, which was similar to the increase in expression observed when the primer to amplify all $S R E B F 1$ isoforms was used; thus, it is likely that the increase in expression of $S R E B F 1$ observed in our study was mostly due to the SREBF1a gene. This is consistent with the observed fact that isoform 1a is expressed in cell culture in vitro (Desvergne et al., 2006).

The large increase in expression of $S R E B F 1$ detected in our study was likely due to the overexpression of the SREBF1 sequence coding for the mature form of SREBP1. Thus, our experiment does not allow us to test whether SREBP1 controls the expression of $S R E B F 1$ in GMEC. However, the SREBP-1c gene is known to have an SREBP1 response element (SRE) in the promoter region as observed in mouse (AmemiyaKudo et al., 2000) and bovine (Lengi and Corl, 2010). We have analyzed the promoter region of the sheep SREBF1a up to 5,000 bp upstream of the transcription starting site (TSS; gene link ENSOARG00000001504) and analyzed the sequence using the free web-based tool LASAGNA (Lee and Huang, 2013) and found a total of 11 SRE motifs, with one motif at $1,547 \mathrm{bp}$ upstream of the TSS being considered very strong. We could not retrieve the promoter region of Capra hircus SREBF1; however, based on the sheep data, it is possible that the upstream region of Capra SREBF1 also presents one or more strong SRE. Based on these data, it is reasonable to expect that the large increases in expression of $S R E B F 1$ and SREBF1a are partly due to the activity of the more-abundant mature SREBP1. The increase in protein abundance of immature SREBP1 in our study was only numerically larger in Ad-nSREBP1 compared with Ad-GFP (Figure 1B), which seems to contrast with the above postulated effect of SREBP1 in its own gene expression in goat. The reason for this apparent contrasting finding is not clear, but it is possible that the effect of SREBP1 on SREBF1 expression is minor in GMEC.

\section{SREBP1 Controls Expression of Milk Fat-Related Genes and Augments TAG Synthesis in GMEC}

It has been demonstrated that $S R E B F 1 c$ overexpression in bovine hepatocytes increases TAG synthesis $(\mathrm{Li}$ et al., 2014b). Similarly, overexpression of SREBF1 in mammary epithelial cells of bovine increased the expression of genes related to fatty acid and TAG synthesis and increased lipid droplet formation and TAG production ( $\mathrm{Li}$ et al., 2014a). Consistent with those previous findings, we observed a significant increase in cellular TAG upon overexpression of nSREBP1 in GMEC. The increase in cellular TAG was consistent with the increase in expression of several genes coding for proteins involved in milk fat synthesis. Interestingly, our data strongly indicate that SREBP1 controls expression of genes in all the metabolic steps for the TAG formation in GMEC. 
The overexpression of nSREBP1 likely increased the generation of acetyl-CoA through greater expression of $A C L Y$ and ACSS2 (Yoshii et al., 2009; Chypre et al., 2012). By providing acetyl-CoA from citrate coming from the tricarboxylic acid cycle, ATP citrate lyase connects glucose/glutamine metabolism to de novo fatty acid synthesis (Hatzivassiliou et al., 2005); however, its role in mammary gland of ruminants does not seem very important, as also demonstrated in goats (Hardwick, 1966).

The positive effect of SREBP1 on de novo fatty acid synthesis is also supported in our study by the increase in expression of $A C A C A$ and FASN, 2 genes coding for rate-limiting enzymes of de novo fatty acid synthesis (Mashima et al., 2009), in agreement with previous studies in mammary tissue of human (Barber et al., 2003), mice (Rudolph et al., 2010), bovine (Oppi-Williams et al., 2013), and goat (Li et al., 2015). The role of SREBP1 in de novo fatty acid synthesis is further supported in our study by the observed increase in expression of IDH1. This gene codes for isocitrate dehydrogenase 1 , a protein generating NADPH that is used by enzymes involved in de novo fatty acid synthesis, such as FASN, and TAG synthesis. Even though the expression of genes coding for proteins involved in de novo fatty acid synthesis was upregulated by overexpression of SREBP1, we cannot conclude that the amount of short- and medium-chain fatty acid was indeed substantially increased, considering the lack of detection of short- and medium-chain fatty acids in our experiment (Table 2). The lack of detection of shortand medium-chain fatty acids was likely caused by the extremely small amount of acetate in the medium used. However, we detected a significant increase in C16:0, indicating that some increase in the de novo synthesis of fatty acids happened in cells with overexpression of SREBP1. It is likely that addition of acetate to the medium would have increased further the production of TAG and production of short- and medium-chain fatty acids.

The large increase in expression of $A C S L 1$, the main enzyme involved in the activation of long-chain fatty acids (LCFA) in bovine mammary, of FABP3, the main LCFA transporter in bovine mammary tissue, and of $S C D 1$, the main desaturation enzyme for LCFA, especially C16:0 and C18:0 (Bionaz and Loor, 2008b), indicated a large increase in the formation of LCFA in Ad-nSREBP1-transfected cells. This was supported by the observed increase in proportions of C18:1 and C16:0 (Table 2). The increase in expression of ELOVL6 due to overexpression of nSREBP1 further supports a role of SREBP1 in the production of LCFA in goat milk. The ELOVL6 gene codes for an enzyme involved in the elongation of LCFA starting from C16:0 and pro- ducing from C18:0 to C26:0 (Jakobsson et al., 2006). No studies have investigated the role of LCFA elongases in mammary tissue of ruminants and their importance remains unclear.

The increased proportions of $\mathrm{C} 16: 0$ and $\mathrm{C} 18: 1$ and decreased proportion of C18:0 in TAG in GMEC overexpressing nSREBP1 is in agreement with the above demonstrated regulatory role of SREBP-1 in de novo fatty acid synthesis, including desaturation by SCD1, which was likely the cause of the decreased proportion of C18:0 and increased proportion of cis-9 C18:1 observed. These data suggest that SREBP1 plays a role in the regulation of the synthesis of MUFA, a role that was previously attributed to peroxisome proliferatoractivated receptor gamma (PPAR $\gamma$; Shi et al., 2013b).

The large increase in expression of INSIG1 and LPIN1 and the decrease in expression of SCAP are interesting findings in our study. The INSIG1 protein can be considered an inhibitor of SREBP1 activity by retaining the inactive SREBP1 in the ER (Bionaz and Loor, 2008b). Lipin 1 also appears to inhibit the nuclear activity of SREBP1, as demonstrated in the mouse (Peterson et al., 2011); in contrast, SCAP is considered a positive activator of SREBP1 (Matsuda et al., 2001). Overall, it appears that the overexpression of SREBP1 induces a negative feedback regulation; thus, SREBP1 controls its own activity.

\section{Potential SREBP1 Target Genes in Goat}

Our study also provides some insights into genes that are targets of SREBP1, albeit indicative rather than definitive, because the increase in expression of genes due to overexpression of nSREBP1 could be caused by a direct effect of SREBP1 but could also be due to secondary effects (i.e., SREBP1 induces expression of other transcription factors that in turn increase the expression of their target genes).

Based on the large increase due to nSREBP1 overexpression, FABP 3 and ACSS2 are likely direct targets of SREBP1. The ACSS2 gene is known to be a direct target of SREBP1 in humans (Luong et al., 2000) and our data confirm this. Our findings are further supported by the observation that expression of FABP3 and ACSS2 increases substantially during lactation in bovine mammary tissue (Bionaz and Loor, 2008a) and decreases dramatically by SREBP1 knockdown and trans-10, cis-12 CLA supplementation in bovine (Kadegowda et al., 2009; Ma and Corl, 2012).

In the current study, the mRNA level of ACSL1 was significantly upregulated by nSREBP1 overexpression in GMEC, in accordance with previous studies (Maxwell et al., 2003; Li et al., 2014a). However, knockdown of SREBP1 by small interfering (si)RNA in Mac-T cells 
and trans-10,cis-12 CLA treatment in bovine kidney cells had no effect on ACSL1 expression (Bionaz et al., 2012; Ma and Corl, 2012). Thus, ACSL1 may not be a direct target of SREBP1, at least in ruminants.

The SCD1 and ELOVL6 genes were upregulated by overexpression of nSREBP1 in our study suggesting that those are SREBP1 target genes. The SCD1 gene is downregulated by trans-10,cis-12 CLA treatment in bovine cells (Peterson et al., 2004; Kadegowda et al., 2009) and its promoter region contains an SRE in bovine (Peterson et al., 2004). Further support comes from a study in which $S R E B F 1$ expression was significantly induced by insulin (Wang et al., 2006).

The LPIN1 and DGAT1 genes were the only ones coding for enzymes responsible for TAG synthesis in mammary tissue (Bionaz and Loor, 2008b). The expression of LPIN1 and DGAT1 genes was upregulated by overexpression of nSREBP1. Previous research has demonstrated that LPIN1 is directly regulated by SREBP1c in hepatoblastoma cells (Ishimoto et al., 2009) and its mRNA expression is downregulated by trans-10,cis-12 CLA in bovine cells (Kadegowda et al., 2009). These previous studies and the upregulation of LPIN1 in our study provide support for LPIN1 being an SREBP1 target gene in goat, at least in vitro; however, LPIN1 is also a known PPAR $\gamma$ target gene (Kadegowda et al., 2009). The expression of DGAT1 is not affected by the inhibition of SREBP1 by trans-10, cis-12 CLA in bovine cells (Kadegowda et al., 2009), implying that DGAT1 may not be a SREBP1 target but the increase in expression observed in our study may be due to SREBP1 activation or increase in expression of a secondary transcription factor. One such factor could be PPAR $\gamma$, because its expression was increased by nSREBP1 overexpression in our study and because activation of this nuclear receptor by a specific synthetic agonist increased the expression of DGAT1 in bovine mammary cells (Kadegowda et al., 2009).

In addition to SREBP1, other transcription factors including INSIG1, SCAP, LXR $\alpha$, and PPAR $\gamma$ can be involved in the transcriptional regulation of milk fat synthesis. Both INSIG1 and SCAP play important roles in the proteolytic process of SREBP; SCAP works as activator, whereas INSIG1 works as an inhibitor of SREBP activity (Yang et al., 2002; Radhakrishnan et al., 2007). The expression of INSIG1 was strongly upregulated by nSREBP1 overexpression, in support of this gene being a target of SREBP1, as previously shown in mouse (Horton et al., 2003), whereas SCAP is not an SREBP1 target (Figure 2).

Liver X receptor $\alpha(\mathrm{LXR} \alpha)$ and PPAR $\gamma$ are liganddependent nuclear receptors with a potential role in controlling milk fat synthesis in bovine (Bionaz and Loor, 2008b; Kadegowda et al., 2009; McFadden and
Corl, 2010). Both nuclear receptors in bovine regulate the expression of SREBF1 (Kadegowda et al., 2009; Lengi and Corl, 2010). In the present study, we observed a dramatic increase of NR1H3 and PPAR $\gamma$ expression by nSREBP1 overexpression, indicating that these nuclear receptors are targets of SREBP1 in goats and supporting cross-talk between transcription factors in the regulation of milk fat synthesis in goat, as previously suggested for bovine (Bionaz and Loor, 2008b). The upregulation of PPAR $\gamma$ in the present study might be a confounding factor in determining SREBP1 target genes because many of the genes measured in the present experiment are putative or demonstrated PPAR $\gamma$ target genes in bovine and goat (Bionaz and Loor, 2008b; Bionaz et al., 2013; Zhao et al., 2014).

\section{CONCLUSIONS}

We observed a large increase in SREBF1 from pregnancy to lactation in goat mammary tissue. Overexpression of nSREBP1 increased the expression of genes related to milk fat synthesis, cellular TAG content, and the amounts of C16:0 and C18:1. A model summarizing the findings from the present experiment is reported in Figure 4 (See color figure in Supplemental Figure S5; http://dx.doi.org/10.3168/jds.2015-9736). Our results provide strong support for SREBP1 being a key regulator for the expression of genes coding for proteins involved in milk fat synthesis, particularly de novo fatty acid and triacylglycerol synthesis, in goat mammary epithelial cells. Furthermore, the positive effect on expression of other transcriptional factors provides support for interactive networks of transcriptional factors involved in the control of milk fat synthesis in goat.

\section{ACKNOWLEDGMENTS}

This research was jointly supported by the Transgenic New Species Breeding Program of China (Beijing, China; 2014ZX08009-051B), National Natural Science Foundation of China (Beijing, China; 31372281), and Special Fund for Agro-scientific Research in the Public Interest (Beijing, China; 201103038).

\section{REFERENCES}

Amemiya-Kudo, M., H. Shimano, T. Yoshikawa, N. Yahagi, A. H. Hasty, H. Okazaki, Y. Tamura, F. Shionoiri, Y. Iizuka, K. Ohashi, J. Osuga, K. Harada, T. Gotoda, R. Sato, S. Kimura, S. Ishibashi, and N. Yamada. 2000. Promoter analysis of the mouse sterol regulatory element-binding protein-1c gene. J. Biol. Chem. 275:3107831085 .

Barber, M. C., A. J. Vallance, H. T. Kennedy, and M. T. Travers. 2003. Induction of transcripts derived from promoter III of the acetyl-CoA carboxylase-alpha gene in mammary gland is associated with recruitment of SREBP-1 to a region of the proximal 
promoter defined by a DNase I hypersensitive site. Biochem. J. 375:489-501.

Bauman, D. E., I. H. Mather, R. J. Wall, and A. L. Lock. 2006. Major advances associated with the biosynthesis of milk. J. Dairy Sci. 89:1235-1243.

Bauman, D. E., J. W. Perfield 2nd, K. J. Harvatine, and L. H. Baumgard. 2008. Regulation of fat synthesis by conjugated linoleic acid: Lactation and the ruminant model. J. Nutr. 138:403-409.

Bionaz, M., S. Chen, M. J. Khan, and J. J. Loor. 2013. Functional role of PPARs in ruminants: Potential targets for fine-tuning metabolism during growth and lactation. PPAR Res. 2013:684159.

Bionaz, M., and J. J. Loor. 2007. Identification of reference genes for quantitative real-time PCR in the bovine mammary gland during the lactation cycle. Physiol. Genomics 29:312-319.

Bionaz, M., and J. J. Loor. 2008a. ACSL1, AGPAT6, FABP3, LPIN1, and SLC27A6 are the most abundant isoforms in bovine mammary tissue and their expression is affected by stage of lactation. J. Nutr. 138:1019-1024.

Bionaz, M., and J. J. Loor. 2008b. Gene networks driving bovine milk fat synthesis during the lactation cycle. BMC Genomics 9:366.

Bionaz, M., B. J. Thering, and J. J. Loor. 2012. Fine metabolic regulation in ruminants via nutrient-gene interactions: Saturated longchain fatty acids increase expression of genes involved in lipid metabolism and immune response partly through PPAR-alpha activation. Br. J. Nutr. 107:179-191.

Briggs, M. R., C. Yokoyama, X. Wang, M. S. Brown, and J. L. Goldstein. 1993. Nuclear protein that binds sterol regulatory element of low density lipoprotein receptor promoter. I. Identification of the protein and delineation of its target nucleotide sequence. J. Biol. Chem. 268:14490-14496.

Brown, M. S., and J. L. Goldstein. 1997. The SREBP pathway: regulation of cholesterol metabolism by proteolysis of a membranebound transcription factor. Cell 89:331-340.

Carcangiu, V., M. C. Mura, C. Daga, S. Luridiana, S. Bodano, G. A. Sanna, M. L. Diaz, and G. Cosso. 2013. Association between SREBP-1 gene expression in mammary gland and milk fat yield in Sarda breed sheep. Meta Gene 1:43-49.

Chen, I. B., V. K. Rathi, D. S. DeAndrade, and P. Y. Jay. 2013 Association of genes with physiological functions by comparative analysis of pooled expression microarray data. Physiol. Genomics 45:69-78.

Chypre, M., N. Zaidi, and K. Smans. 2012. ATP-citrate lyase: A minireview. Biochem. Biophys. Res. Commun. 422:1-4.

Clegg, R. A., M. C. Barber, L. Pooley, I. Ernens, Y. Larondelle, and M. T. Travers. 2001. Milk fat synthesis and secretion: molecular and cellular aspects. Livest. Prod. Sci. 70:3-14.

Desvergne, B., L. Michalik, and W. Wahli. 2006. Transcriptional regulation of metabolism. Physiol. Rev. 86:465-514.

Eberlé, D., B. Hegarty, P. Bossard, P. Ferre, and F. Foufelle. 2004. SREBP transcription factors: Master regulators of lipid homeostasis. Biochimie 86:839-848.

Espenshade, P. J. 2006. SREBPs: Sterol-regulated transcription factors. J. Cell Sci. 119:973-976.

Hardwick, D. C. 1966. The fate of acetyl groups derived from glucose in the isolated perfused goat udder. Biochem. J. 99:228-231.

Harvatine, K. J., and D. E. Bauman. 2006. SREBP1 and thyroid hormone responsive spot 14 (S14) are involved in the regulation of bovine mammary lipid synthesis during diet-induced milk fat depression and treatment with CLA. J. Nutr. 136:2468-2474.

Harvatine, K. J., Y. R. Boisclair, and D. E. Bauman. 2009. Recent advances in the regulation of milk fat synthesis. Animal 3:40-54.

Hatzivassiliou, G., F. Zhao, D. E. Bauer, C. Andreadis, A. N. Shaw, D. Dhanak, S. R. Hingorani, D. A. Tuveson, and C. B. Thompson. 2005. ATP citrate lyase inhibition can suppress tumor cell growth. Cancer Cell 8:311-321.

He, T. C., S. Zhou, L. T. da Costa, J. Yu, K. W. Kinzler, and B. Vogelstein. 1998. A simplified system for generating recombinant adenoviruses. Proc. Natl. Acad. Sci. USA 95:2509-2514.

Horton, J. D. 2002. Sterol regulatory element-binding proteins: Transcriptional activators of lipid synthesis. Biochem. Soc. Trans. 30:1091-1095
Horton, J. D., N. A. Shah, J. A. Warrington, N. N. Anderson, S. W. Park, M. S. Brown, and J. L. Goldstein. 2003. Combined analysis of oligonucleotide microarray data from transgenic and knockout mice identifies direct SREBP target genes. Proc. Natl. Acad. Sci. USA 100:12027-12032

Ishimoto, K., H. Nakamura, K. Tachibana, D. Yamasaki, A. Ota, K. Hirano, T. Tanaka, T. Hamakubo, J. Sakai, T. Kodama, and T. Doi. 2009. Sterol-mediated regulation of human lipin 1 gene expression in hepatoblastoma cells. J. Biol. Chem. 284:22195-22205.

Jakobsson, A., R. Westerberg, and A. Jacobsson. 2006. Fatty acid elongases in mammals: Their regulation and roles in metabolism. Prog. Lipid Res. 45:237-249.

Kadegowda, A. K., M. Bionaz, L. S. Piperova, R. A. Erdman, and J. J. Loor. 2009. Peroxisome proliferator-activated receptor-gamma activation and long-chain fatty acids alter lipogenic gene networks in bovine mammary epithelial cells to various extents. J. Dairy Sci. 92:4276-4289.

Lee, C., and C. H. Huang. 2013. LASAGNA: A novel algorithm for transcription factor binding site alignment. BMC Bioinformatics 14:108.

Lengi, A. J., and B. A. Corl. 2010. Short communication: Identification of the bovine sterol regulatory element binding protein$1 c$ promoter and its activation by liver X receptor. J. Dairy Sci. 93:5831-5836.

Li, J., J. Luo, H. Xu, M. Wang, J. Zhu, H. Shi, A. B. Haile, H. Wang, and Y. Sun. 2015. Fatty acid synthase promoter: Characterization, and transcriptional regulation by sterol regulatory element binding protein-1 in goat mammary epithelial cells. Gene 561:157-164.

Li, N., F. Zhao, C. Wei, M. Liang, N. Zhang, C. Wang, Q. Z. Li, and X. J. Gao. 2014a. Function of SREBP1 in the milk fat synthesis of dairy cow mammary epithelial cells. Int. J. Mol. Sci. 15:1699817013.

Li, X., Y. Li, W. Yang, C. Xiao, S. Fu, Q. Deng, H. Ding, Z. Wang, G. Liu, and X. Li. 2014b. SREBP-1c overexpression induces triglycerides accumulation through increasing lipid synthesis and decreasing lipid oxidation and VLDL assembly in bovine hepatocytes. J. Steroid Biochem. Mol. Biol. 143:174-182.

Lin, X. Z., J. Luo, L. P. Zhang, W. Wang, H. B. Shi, and J. J. Zhu. 2013. MiR-27a suppresses triglyceride accumulation and affects gene mRNA expression associated with fat metabolism in dairy goat mammary gland epithelial cells. Gene 521:15-23.

Luong, A., V. C. Hannah, M. S. Brown, and J. L. Goldstein. 2000. Molecular characterization of human acetyl-CoA synthetase, an enzyme regulated by sterol regulatory element-binding proteins. J. Biol. Chem. 275:26458-26466.

Ma, L., and B. A. Corl. 2012. Transcriptional regulation of lipid synthesis in bovine mammary epithelial cells by sterol regulatory element binding protein-1. J. Dairy Sci. 95:3743-3755.

Mashima, T., H. Seimiya, and T. Tsuruo. 2009. De novo fatty-acid synthesis and related pathways as molecular targets for cancer therapy. Br. J. Cancer 100:1369-1372.

Matsuda, M., B. S. Korn, R. E. Hammer, Y. A. Moon, R. Komuro, J. D. Horton, J. L. Goldstein, M. S. Brown, and I. Shimomura 2001. SREBP cleavage-activating protein (SCAP) is required for increased lipid synthesis in liver induced by cholesterol deprivation and insulin elevation. Genes Dev. 15:1206-1216.

Maxwell, K. N., R. E. Soccio, E. M. Duncan, E. Sehayek, and J. L. Breslow. 2003. Novel putative SREBP and LXR target genes identified by microarray analysis in liver of cholesterol-fed mice. J. Lipid Res. 44:2109-2119.

McFadden, J. W., and B. A. Corl. 2010. Activation of liver X receptor (LXR) enhances de novo fatty acid synthesis in bovine mammary epithelial cells. J. Dairy Sci. 93:4651-4658.

Moore, J. H., and W. W. Christie. 1979. Lipid metabolism in the mammary gland of ruminant animals. Prog. Lipid Res. 17:347-395.

Nohturfft, A., M. S. Brown, and J. L. Goldstein. 1998. Topology of SREBP cleavage-activating protein, a polytopic membrane protein with a sterol-sensing domain. J. Biol. Chem. 273:17243-17250.

Oppi-Williams, C., J. K. Suagee, and B. A. Corl. 2013. Regulation of lipid synthesis by liver $\mathrm{X}$ receptor alpha and sterol regulatory 
element-binding protein 1 in mammary epithelial cells. J. Dairy Sci. 96:112-121.

Peterson, D. G., E. A. Matitashvili, and D. E. Bauman. 2004. The inhibitory effect of trans-10, cis-12 CLA on lipid synthesis in bovine mammary epithelial cells involves reduced proteolytic activation of the transcription factor SREBP-1. J. Nutr. 134:2523-2527.

Peterson, T. R., S. S. Sengupta, T. E. Harris, A. E. Carmack, S. A. Kang, E. Balderas, D. A. Guertin, K. L. Madden, A. E. Carpenter, B. N. Finck, and D. M. Sabatini. 2011. mTOR complex 1 regulates lipin 1 localization to control the SREBP pathway. Cell 146:408-420.

Radhakrishnan, A., Y. Ikeda, H. J. Kwon, M. S. Brown, and J. L. Goldstein. 2007. Sterol-regulated transport of SREBPs from endoplasmic reticulum to Golgi: Oxysterols block transport by binding to Insig. Proc. Natl. Acad. Sci. USA 104:6511-6518.

Ramakers, C., J. M. Ruijter, R. H. L. Deprez, and A. F. M. Moorman. 2003. Assumption-free analysis of quantitative real-time polymerase chain reaction (PCR) data. Neurosci. Lett. 339:62-66.

Rudolph, M. C., J. Monks, V. Burns, M. Phistry, R. Marians, M. R. Foote, D. E. Bauman, S. M. Anderson, and M. C. Neville. 2010 Sterol regulatory element binding protein and dietary lipid regulation of fatty acid synthesis in the mammary epithelium. Am. J. Physiol. Endocrinol. Metab. 299:E918-E927.

Sato, R. 2010. Sterol metabolism and SREBP activation. Arch. Biochem. Biophys. 501:177-181.

Shi, H., J. Luo, J. Zhu, J. Li, Y. Sun, X. Lin, L. Zhang, D. Yao, and H. Shi. 2013a. PPAR gamma regulates genes involved in triacylglycerol synthesis and secretion in mammary gland epithelial cells of dairy goats. PPAR Res. 2013:310948.

Shi, H. B., J. Luo, D. W. Yao, J. J. Zhu, H. F. Xu, H. P. Shi, and J. J. Loor. 2013b. Peroxisome proliferator-activated receptor-gamma stimulates the synthesis of monounsaturated fatty acids in dairy goat mammary epithelial cells via the control of stearoyl-coenzyme A desaturase. J. Dairy Sci. 96:7844-7853.

Shi, H. B., W. S. Zhao, J. Luo, D. W. Yao, Y. T. Sun, J. Li, H. P. Shi, and J. J. Loor. 2014. Peroxisome proliferator-activated receptor gamma1 and gamma2 isoforms alter lipogenic gene networks in goat mammary epithelial cells to different extents. J. Dairy Sci. 97:5437-5447.

Tontonoz, P., J. B. Kim, R. A. Graves, and B. M. Spiegelman. 1993. ADD1: A novel helix-loop-helix transcription factor associated with adipocyte determination and differentiation. Mol. Cell. Biol. $13: 4753-4759$ van Dorland, H. A., S. Richter, I. Morel, M. G. Doherr, N. Castro, and R. M. Bruckmaier. 2009. Variation in hepatic regulation of metabolism during the dry period and in early lactation in dairy cows. J. Dairy Sci. 92:1924-1940.

Vandesompele, J., K. De Preter, F. Pattyn, B. Poppe, N. Van Roy, A. De Paepe, and F. Speleman. 2002. Accurate normalization of real-time quantitative RT-PCR data by geometric averaging of multiple internal control genes. Genome Biol. 3:RESEARCH0034.

Wang, W., J. Luo, Y. Zhong, X. Z. Lin, H. B. Shi, J. J. Zhu, J. Li, Y. T. Sun, and W. S. Zhao. 2012. Goat liver X receptor alpha, molecular cloning, functional characterization and regulating fatty acid synthesis in epithelial cells of goat mammary glands. Gene 505:114-120.

Wang, X., M. R. Briggs, X. Hua, C. Yokoyama, J. L. Goldstein, and M. S. Brown. 1993. Nuclear protein that binds sterol regulatory element of low density lipoprotein receptor promoter. II. Purification and characterization. J. Biol. Chem. 268:14497-14504.

Wang, Y., D. Botolin, J. Xu, B. Christian, E. Mitchell, B. Jayaprakasam, M. G. Nair, J. M. Peters, J. V. Busik, L. K. Olson, and D. B. Jump. 2006. Regulation of hepatic fatty acid elongase and desaturase expression in diabetes and obesity. J. Lipid Res. 47:2028-2041.

Yang, T., P. J. Espenshade, M. E. Wright, D. Yabe, Y. Gong, R. Aebersold, J. L. Goldstein, and M. S. Brown. 2002. Crucial step in cholesterol homeostasis: Sterols promote binding of SCAP to INSIG-1, a membrane protein that facilitates retention of SREBPs in ER. Cell 110:489-500.

Yokoyama, C., X. D. Wang, M. R. Briggs, A. Admon, J. Wu, X. X. Hua, J. L. Goldstein, and M. S. Brown. 1993. Srebp-1, a basichelix-loop-helix-leucine zipper protein that controls transcription of the low density lipoprotein receptor gene. Cell 75:187-197.

Yoshii, Y., T. Furukawa, H. Yoshii, T. Mori, Y. Kiyono, A. Waki, M. Kobayashi, T. Tsujikawa, T. Kudo, H. Okazawa, Y. Yonekura, and Y. Fujibayashi. 2009. Cytosolic acetyl-CoA synthetase affected tumor cell survival under hypoxia: The possible function in tumor acetyl-CoA/acetate metabolism. Cancer Sci. 100:821-827.

Zhao, W., M. Bionaz, J. Luo, A. Hosseini, and J. J. Loor. 2014. Long chain fatty acids alter expression of genes involved in lipid metabolism in goat mammary epithelial cells partly through PPARgamma. J. Dairy Sci. 97(E-Suppl. 1):321. (Abstr.) 